Pacific Journal of Mathematics

A CLASS OF DIVISIBLE MODULES 


\title{
A CLASS OF DIVISIBLE MODULES
}

\author{
MARK L. TEPLY
}

The $\mathscr{T}$-divisible $R$-modules are defined in terms of a hereditary torsion theory of modules over an associative ring $R$ with identity element. In the special case where $\mathscr{T}$ is the usual torsion class of modules over a commutative integral domain, the class of $\mathscr{T}$-divisible modules is precisely the class of divisible modules $M$ such that every nonzero homomorphic image of $M$ has a nonzero $h$-divisible submodule. In general, if $\mathscr{T}$ is a stable hereditary torsion class, the class of $\mathscr{T}$-divisible modules satisfies many of the traditional properties of divisible modules over a commutative integral domain. This is especially true when $\mathscr{T}$ is Goldie's torsion class $\mathscr{G}$. For suitable $\mathscr{T}$, the splitting of all $\mathscr{T}$-divisible modules is equivalent to h.d. $Q_{\mathscr{T}} \leqq 1$, where $Q_{\mathscr{T}}$ is the ring of quotients naturally associated with $\mathscr{T}$. Generalizations of Dedekind domains are studied in terms of $\mathscr{T}$-divisibility.

1. Notation, terminology, and preliminary results. In this paper, all rings $R$ are associative rings with identity element, and all modules are unitary left $R$-modules. ${ }_{R}-\mathscr{l}$ denotes the category of all left $R$-modules. $E(M)$ denotes the injective envelope of $M \in_{R} \mathscr{C}$. In homological expressions, the " $R$ " will be omitted for convenience in printing (e.g. Ext ${ }_{R}^{1}=\operatorname{Ext}^{1}$ and h.d. ${ }_{R} Q=$ h.d. $Q$ ).

Following S.E. Dickson [4], we call a nonempty subclass $\mathscr{T}$ of ${ }_{n}$ ll a torsion class if $\mathscr{T}$ is closed under factors, extensions, and arbitrary direct sums. $\mathscr{T}$ is called hereditary if it is closed under submodules. Modules in $\mathscr{T}$ are called torsion. Every torsion class $\mathscr{T}$ determines in every $A \in_{R} \mathscr{C}$ a unique maximal torsion submodule $\mathscr{T}(A)$. $\mathscr{T}(A)$ is called the torsion submodule of $A$, and $\mathscr{T}(A / \mathscr{T}(A))=0$. Modules in $\mathscr{F}=\left\{A \in_{R} \mathscr{C l} \mid \mathscr{T}(A)=0\right\}$ are called torsionfree, and the torsionfree class $\mathscr{F}$ is closed under submodules, extensions, and direct products. $\mathscr{T}$ is hereditary if and only if $\mathscr{F}$ is closed under injective envelopes.

Throughout this paper, $\mathscr{T}^{-}$will denote a hereditary torsion class and $\mathscr{F}$ will denote the torsionfree class corresponding to $\mathscr{T}$; hence $(\mathscr{T}, \mathscr{F})$ is a hereditary torsion theory [4], [10], and [17]. Each such $(\mathscr{T}, \mathscr{F})$ is uniquely associated with a topologizing and idempotent filter $F(\mathscr{T})=\{I \mid I \leqq R$ and $R / I \in \mathscr{T}\}$ of left ideals of $R$.

In [5] the right derived functors for a hereditary torsion class $\mathscr{T}$ are examined. These derived functors are given by

$$
R_{\mathcal{J}}^{\circ}(A)=\mathscr{T}(A), R^{1}(A)=\mathscr{T}(E(A) / A) / \frac{\mathscr{T}(E(A))+A}{A},
$$


and $R_{\mathscr{S}}^{n}(A)=R_{\mathscr{S}}^{n-1}(E(A) / A)$ for $n \geqq 2$. If $A$ is injective, then $R_{\mathscr{S}}^{n}(A)=0$ for $n \geqq 1$. If $A \in \mathscr{F}$, then $R_{\mathscr{T}}^{1}(A)=(\mathscr{T} E(A) / A)$. A module $M$ is called $\mathscr{T}$-injective ([8], [9], and [15]) if $\operatorname{Ext}^{1}(T, M)=0$ for all $T \in \mathscr{T}$. (Note: in [14] $\mathscr{T}$-injective modules are called $F(\mathscr{T})$-divisible.) This happens exactly when $\mathscr{T}(E(M) / M)=0$ (see [8]). Hence a module $A \in \mathscr{F}$ is $\mathscr{T}$-injective if and only if $R_{\mathscr{T}}^{1}(A)=0$. If

$$
0 \longrightarrow A \longrightarrow B \longrightarrow C \longrightarrow 0
$$

is any short exact sequence, then so is the induced sequence $0 \rightarrow \mathscr{I}(A) \rightarrow$ $\mathscr{T}(B) \rightarrow \mathscr{T}(C) \rightarrow R_{\mathscr{T}}^{1}(A) \rightarrow R_{\mathscr{T}}^{1}(B) \rightarrow R_{\mathscr{T}}^{1}(C) \rightarrow R_{\mathscr{T}}^{2}(A) \rightarrow \cdots$.

A torsion class $\mathscr{T}$ is called stable if $\mathscr{T}$ is closed under injective envelopes. $\quad R_{\mathscr{T}}^{1}(T)=0$ for all $T \in \mathscr{T}$; and if $\mathscr{T}$ is stable, then $R_{\mathscr{S}}^{n}(T)=0$ for all $T \in \mathscr{T}$ and for all $n \geqq 1$. This and the long exact sequence in the preceding paragraph imply that if $\mathscr{T}$ is stable and $B \in_{R} \mathscr{C l}$, then $R_{\mathscr{T}}^{n}(B)=0$ if and only if $R_{\mathscr{S}}^{n}(B / \mathscr{T}(B))=0$.

The submodule $E_{\mathcal{F}}(M)$ of $E(M)$ is the (unique) largest module satisfying $M \subseteq E_{\mathcal{J}}(M) \subseteq E(M)$ and $E_{\mathscr{T}}(M) / M \in \mathscr{T}$. We call $E_{\mathscr{T}}(M)$ the $\mathscr{T}$-injective envelope of $M . E_{\mathscr{T}}(M)$ exists [14] and is $\mathscr{T}$-injective. If $R \in \mathscr{F}$, then we will use $Q_{\mathscr{T}}$ to denote $E_{\mathscr{T}}(R) . Q_{\mathscr{T}}$ has a ring structure [8] and has received attention in a large number of papers (e.g.[7], [8], [9], and [15]).

A module $M$ is said to split if $\mathscr{T}(M)$ is a direct summand of $M$.

We now state a lemma giving some properties of $\mathscr{T}$-injective modules.

Lemma 1.1. (1) $\mathscr{T}$ is stable if and only if every $\mathscr{T}$-injective modules splits.

(2) A direct summand of a $\mathscr{T}$-injective module is $\mathscr{T}$-injective.

(3) If $A$ is a $\mathscr{T}$-injective module and if $\theta: A \rightarrow B$ is an epimorphism, then $\theta^{-1}(\mathscr{T}(B))=\{x \in A \mid \theta(x) \in \mathscr{T}(B)\}$ is $\mathscr{T}$-injective.

(4) If $\mathscr{T}$ is stable, then every $\mathscr{T}$-injective module in $\mathscr{T}$ is injective.

Proof. (1) If $\mathscr{T}$ is stable and $A$ is $\mathscr{T}$-injective, then $E(A) / A \in \mathscr{F}$; so $\mathscr{T}(E(A)) \cong A$. By [2, Prop. 2.1], $E(A)=\mathscr{T}(E(A)) \oplus F$ for some $F \in \mathscr{F}$. Hence $A=\mathscr{T}(E(A)) \oplus(F \cap A)$. Since $\mathscr{T}(E(A))=\mathscr{T}(A)$ in this case, then $A$ splits.

The converse is immediate from [2, Prop. 2.1].

(2) This is well-known and straight forward to prove.

(3) Note that

$$
E_{-} \frac{\left(\theta^{-1}(\mathscr{T}(B))\right)}{\theta^{-1}(\mathscr{T}(B))} \leqq \frac{A}{\theta^{-1}(\mathscr{T}(B))} \cong \frac{\theta(A)}{\theta \theta^{-1}(\mathscr{T}(B))}=\frac{B}{\mathscr{T}(B)} \in \mathscr{F} .
$$


So by the definition of $\mathscr{T}$-injective envelope, $E_{-}\left(\theta^{-1}(\mathscr{T}(B))\right)=$ $\theta^{-1}(\mathscr{T}(B))$.

(4) Let $T \in \mathscr{T}$ be $\mathscr{T}$-injective; so $E(T) \in \mathscr{T}$ and $E(T) / T \in \mathscr{F}$. Since $\mathscr{T}$ is closed under homomorphic images, $E(T) / T \in \mathscr{T} \cap \mathscr{F}=\{0\}$; thus $E(T)=T$.

We now turn our attention to an important special torsion theory. The class $\mathscr{G}$ of Goldie torsion modules is the smallest class containing all isomorphic copies of all factor modules $A / B$, where $B$ is an essential submodule of $A$ (see [1], [2], [16], and [17]). The corresponding Goldie torsionfree class $\mathscr{N}$ is exactly the class of nonsingular modules. $\mathscr{S}$ is hereditary and stable; if $R \in \mathscr{N}$, then $\mathscr{G}$ is precisely the class of singular modules. So if $R$ is a commutative integral domain, then $\mathscr{G}$ and $\mathscr{N}$ coincide with the usual torsion and torsionfree classes, respectively. This makes $(\mathscr{C}, \mathcal{N}$ ) a "natural" torsion theory to consider when one is trying to generalize the usual results about torsion modules over an integral domain. Moreover, the $\mathscr{C}$ injective modules are just the injective modules; and if $R \in \mathscr{N}$, then $Q \cong E(R)$ and the filter $F(\mathscr{G})$ is the set of essential left ideals of $R$.

2. T-divisible modules. Historically the class of divisible modules has been very useful in studying modules over a commutative integral domain. Frequently subclasses of the class of divisible modules have also yielded interesting results (e.g. see [11] and [12]). One such subclass studied by Matlis is the class of $h$-divisible modules, i.e., those modules which are homomorphic images of injective modules. A class related to the $h$-divisible modules is the class of divisible modules $M$ such that every nonzero homomorphic image of $M$ has a nonzero $h$-divisible submodule. For reasons indicated later, we refer to this latter class as the $\mathscr{C}$-divisible modules. We shall show that not only does the class of -divisible modules satisfy many interesting properties but also it allows us to "smooth out" some of Matlis" results. For example, Matlis proves [11, Cor. 2.6] that every divisible module splits if and only if (1) h.d. $Q \leqq 1$ (where $Q$ is the quotient field of $R$ ) and (2) $T \cong \operatorname{Ext}^{1}(Q / R, T)$ for every torsion divisible module $T$ with 0 as its only $h$-divisible submodule. We remove condition (2) by only considering $\mathscr{C}$-divisible modules: every $\mathscr{G}$-divisible module splits if and only if h.d. $Q \leqq 1$ (see Corollary 4.6).

However, we will not limit our investigation to modules over a commutative integral domain. We are able to state our entire theory in terms of hereditary torsion theories $(\mathscr{T}, \mathscr{F}$ ) of modules over an associative ring $R$ with identity element.

To do this, we begin by defining subclasses $\mathscr{C}_{\alpha}$ of ${ }_{R} \mathscr{C l}$ for each ordinal number $\alpha$. 
$\mathscr{C}_{1}=\left\{M \in_{R} \mathscr{C l} \mid M\right.$ is a homomorphic image of a direct sum of $\mathscr{T}$-injective modules\}. If $\alpha$ is not a limit ordinal, then $\mathscr{C}_{\alpha}=$ $\left\{M \in_{R} \mathscr{M l} \mid \exists N \subseteq M\right.$ such that $N \in \mathscr{C}_{\alpha-1}$ and $\left.M / N \in \mathscr{C}_{1}\right\}$. If $\alpha$ is a limit ordinal, then $\mathscr{C}_{\alpha}=\left\{M \in_{R} \mathscr{M} \mid M=\cup_{\beta<\alpha} N_{\beta}\right.$ where $N_{\beta} \in \mathscr{C}_{\beta}$ for $\left.\beta<\alpha\right\}$. This transfinite definition is similar to the construction of a Loewy series [6]. Clearly $\mathscr{C}_{\beta} \leqq \mathscr{C}_{\alpha}$ for $\beta \leqq \alpha$. It is straight forward to show that each $\mathscr{C}_{\alpha}$ is closed under homomorphic images and direct sums.

If $R$ is a commutative integral domain and if the torsion class is taken to be $\mathscr{G}$, it follows that each $\mathscr{C}_{\alpha}$ is a subclass of the (usual) class of divisible modules. Also, over a commutative integral domain, any divisible module in $\mathscr{N}$ is injective.

These two facts, plus the fact that each $\mathscr{C}_{\alpha}$ depends on $\mathscr{T}$,

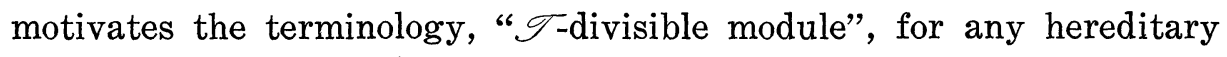
torsion theory $(\mathscr{T}, \mathscr{F})$ over any ring.

Definition. A module $M$ is called $\mathscr{T}$-divisible if and only if (i) $M / \mathscr{T}(M)$ is $\mathscr{T}$-injective and (ii) $\mathscr{T}(M) \in \mathscr{C}_{\alpha}$ for some ordinal $\alpha$. $\mathscr{D} \mathscr{T}$ will denote the class of all $\mathscr{T}$-divisible modules.

ExAmple 2.1. If $R$ is a commutative integral domain, then a module $M$ is $\mathscr{G}$-divisible if and only if (a) $M$ is divisible and (b) every nonzero homomorphic image of $M$ has a nonzero $h$-divisible submodule.

Let $M \in \mathscr{D} \mathscr{G}$. From the construction of the classes $\mathscr{C}_{\alpha}$, the closure properties of divisible modules, and the definition of $\mathscr{G}$-divisible, it follows that $M$ is divisible. Let $\theta: M \rightarrow X$ be an epimorphism. If ker $\theta$ does not contain $\mathscr{C}(M)$, then it follows from (ii) that $(\mathscr{C}(M)+$ $\operatorname{ker} \theta) / \operatorname{ker} \theta$ contains an $h$-divisible submodule. If $\operatorname{ker} \theta \supseteqq \mathscr{G}(M)$, then $X$ is a homomorphic image of $M / \mathscr{C}(M)$, and hence $X$ is $h$-divisible by (i).

Conversely, let $M$ satisfy (a) and (b). Since $M / \mathscr{C}(M)$ is torsionfree and divisible, then $M / \mathscr{G}(M)$ is injective by [3, VII, Prop. 1.3]; so (i) holds.

Since any nonzero $\mathscr{C}$-torsionfree injective module is a direct sum of copies of the quotient field $Q$, then $M$ contains a maximal $\mathscr{C}$ torsionfree injective module $B$ (possibly $B=0$ ). Set $M=A \oplus B$. By (b) $A$ contains an $h$-divisible submodule $H_{1}$. From [11, Theorem 1.1] and [3, VII. Prop. 1.3), it follows that $H_{1}=\mathscr{G}\left(H_{1}\right) \oplus F$ with $F \in \mathscr{N}$ injective. By the definition of $B, F=0$. Hence $H_{1} \subseteq \mathscr{G}(A)=\mathscr{G}(M)$. Using similar reasoning on $A / H_{1}$, we can find $H_{2} \subseteq A$ such that $H_{2} / H_{1}$ is an $h$-divisible submodule of $\mathscr{G}\left(A / H_{1}\right)=\mathscr{C}(A) / H_{1}$. Thus $H_{2} \in \mathscr{C}_{2} \cap \mathscr{G}$. If $\beta$ is a limit ordinal and $H_{\gamma} \in \mathscr{C}_{r} \cap \mathscr{G}$ has been defined for all $\gamma<\beta$, define $H_{\beta}=\cup_{r<\beta} H_{r}$; then $H_{\beta} \in \mathscr{C}_{\beta} \cap \mathscr{G}$. Proceeding by transfinite 
induction, the reader can now easily show that there exists $H_{\alpha} \in \mathscr{C}_{\alpha}$ such that $H_{\alpha}=\mathscr{C}(A)=\mathscr{C}(M)$; so (ii) holds.

EXAMPLE 2.2. The homomorphic image of a $\mathscr{T}$-injective module need not be $\mathscr{T}$-divisible.

Let $K$ be a field, let $R=K[x, y]$ (commutative), and let $M=$ $(x, y)$. Define

$$
F\left(\mathscr{T}_{M}\right)=\left\{I \subseteq R \mid M^{n} \sqsubseteq I \text { for some } n\right\} .
$$

Then [15, p. 40, Ex. 3] and [8, Theorem 4.5] show that there exists a $\mathscr{T}_{M}$-injective module $E \in \mathscr{F}_{M}$ with a homomorphic image $E / E^{\prime} \in \mathscr{F}_{M}$ such that $E / E^{\prime}$ is not $\mathscr{T}_{M}$-injective. Thus $E / E^{\prime}$ is not $\mathscr{T}_{M}$-divisible.

For later use, we note that since $R$ is a commutative Noetherian ring, then $\mathscr{T}_{y}$ is stable by [15, Prop. 5.12].

Our first result shows that the class of $\mathscr{T}$-divisible modules satisfies some traditional properties [3, p. 128] of modules over a commutative integral domain.

Proposition 2.3. (1) If $\mathscr{G}$ is stable, then every injective module is $\mathscr{T}$-divisible.

(2) If $\mathscr{T}$ is stable, then every $\mathscr{T}$-injective module is $\mathscr{G}$-divisible.

(3) Every $\mathscr{T}$-injective module in $\mathscr{T}$ is $\mathscr{T}$-divisible.

(4) Every $\mathscr{T}$-divisible module in $\mathscr{F}$ is $\mathscr{T}$-injective.

Proof. (1) and (2). Since every injective module is $\mathscr{G}$-injective, it is sufficient to show that every $\mathscr{G}$-injective module $A$ is $\mathscr{G}$-divisible. By Lemma 1.1 (1), $A$ splits, and hence $\mathscr{T}(A)$ and $A / \mathscr{T}(A)$ are both $\mathscr{S}$-injective by Lemma 1.1 (2). Thus $A$ is $\mathscr{T}$-divisible.

(3) and (4) follow from the definition of a $\mathscr{S}$-divisible module.

The next three propositions investigate the closure properties of the class $\mathscr{D} \mathscr{Y}^{-}$of $\mathscr{T}$-divisible modules.

Proposition 2.4. If $\mathscr{T}$ is stable, then the following statements are true.

(1) $\mathscr{D S}$ is closed under injective envelopes.

(2) $\mathscr{D} T$ is closed under $\mathscr{T}$-injective envelopes.

(3) $\mathscr{D} \mathscr{T}$ is closed under direct summands.

(4) $\mathscr{D S}$ is closed under extensions.

(5) $\mathscr{D S}$ is closed under finite direct sums.

Proof. (1) follows from Proposition 2.3 (1).

(2) follows from Proposition 2.3 (2).

(3) Suppose that $A=B \oplus C$ and that $A \in \mathscr{Q} \mathscr{T}$. Then $\mathscr{T}(B)=$ $\pi\left(\mathscr{S}^{-}(A)\right)$, where $\pi$ is the projection map from $A$ to $B$. Since $\mathscr{G}(A) \in \mathscr{C}_{\alpha}$ for some ordinal $\alpha$ and since $\mathscr{C}_{\alpha}$ is closed under homomorphic images, 
then $\mathscr{T}(B) \in \mathscr{C}_{\alpha}$ also. Since $A \in \mathscr{D} \mathscr{T}$, then $A / \mathscr{T}(A)$ is $\mathscr{T}$-injective. Since $A / \mathscr{T}(A) \cong(B / \mathscr{T}(B)) \oplus(C / \mathscr{T}(C))$, then $B / \mathscr{T}(B)$ is $\mathscr{T}$-injective by Lemma 1.1 (2). Therefore $B$ is $\mathscr{T}$-divisible.

(4) Let $A, B \in \mathscr{D} \mathscr{T}$, and let

$$
0 \longrightarrow A \longrightarrow X \longrightarrow B \longrightarrow 0
$$

be an exact sequence. Since $A, B \in \mathscr{D} \mathscr{T}$, the induced sequence

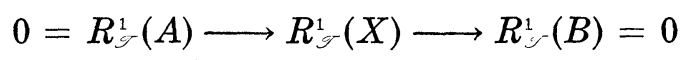

is exact. Since $\mathscr{T}$ is stable, it follows from the preceding sequence that $R_{\mathscr{T}}^{1}(X / \mathscr{T}(X))=0$. Hence $X / \mathscr{T}(X)$ is $\mathscr{T}$-injective.

Since $A, B \in \mathscr{D} \mathscr{T}$, then $\mathscr{T}(A) \in \mathscr{C}_{\alpha}$ and $\mathscr{T}(B) \in \mathscr{C}_{\beta}$ for some ordinals $\alpha$ and $\beta$. Since $R_{\mathscr{S}}^{1}(A)=0$, the sequence

$$
0 \longrightarrow \mathscr{T}(A) \longrightarrow \mathscr{T}(X) \longrightarrow \mathscr{T}\left(B \longrightarrow R_{\mathscr{T}}^{1}(A)=0\right.
$$

is exact. It is straight forward to verify from this sequence that $\mathscr{T}(X) \in \mathscr{C}_{\alpha+\beta}$.

(5) follows from (4) by induction.

If $\mathscr{T}$ is a hereditary torsion class in ${ }_{R} \mathscr{C}$, then a ring $R$ is said to have $\mathscr{T}$-gl. $\operatorname{dim} R=n$ if $R^{n+1}(M)=0$ for all $M \in_{R} \mathscr{M l}$. In [17] conditions equivalent to $\mathscr{T}$-gl. $\operatorname{dim} R=0$ are given. If $\mathscr{T}$ is stable, then the next result gives a characterization of $\mathscr{T}-\mathrm{gl} . \operatorname{dim} R \leqq 1$.

Proposition 2.5. If $\mathscr{T}$ is stable, then the following statements are equivalent.

(1) $\mathscr{D} \mathscr{T}$ is closed under homomorphic images.

(2) $\mathscr{T}$-gl. $\operatorname{dim} R \leqq 1$.

(3) For any $\mathscr{T}$-injective module $A$ and any epimorphism $\theta: A \rightarrow B$ such that $B \in \mathscr{F}, B$ is $\mathscr{T}$-injective.

(4) For any $\mathscr{T}$-injective module $A \in \mathscr{F}$ and any epimorphism $\theta: A \rightarrow B$ such that $B \in \mathscr{F}, B$ is $\mathscr{T}$-injective.

REMARKs. (i) Additional conditions equivalent to (4) are given in [8, Theorem 4.5].

(ii) The equivalence of (2) and (3) is due to C. Megibben, who communicated this equivalence to the author in a personal letter (Jan., 1971). The author wishes to thank Professor Megibben for sending him the result. Moreover, the proof of the equivalence of (2) and (3) does not require that $\mathscr{T}$ be stable.

(iii) The interesting case in Proposition 2.5 is when $\mathscr{T}-\mathrm{gl} . \operatorname{dim} R=1$. For if $R \in \mathscr{F}$ and $\mathscr{T}$-gl. $\operatorname{dim} R=0$, then by [17, Theorem 3.1 (3)] every module in ${ }_{R} \mathscr{C l}$ is also in $\mathscr{F}$.

(iv) In Example 2.2, $\mathscr{T}_{M}$-gl. $\operatorname{dim} R>1$, and $\mathscr{T}_{M}$ is stable; so not every hereditary torsion theory satisfies Proposition 2.5. 
Proof of Proposition 2.5. (1) $\Rightarrow(2)$. It follows from the exactness of the sequence

$$
R_{\digamma}^{1}(E(M) / M) \longrightarrow R_{\digamma}^{2}(M) \longrightarrow R_{\smile}^{2}(E(M))=0
$$

that it is sufficient to show $R_{\mathscr{T}}^{1}(E(M) / M)=0$. Since $\mathscr{T}$ is stable, Proposition 2.3 (1) implies $E(M)$ is $\mathscr{T}$-divisible. Set $X=E(M) / M$. By (1), $X / \mathscr{T}(X) \in \mathscr{F}$ is $\mathscr{T}$-divisible; hence $X / \mathscr{T}(X)$ is $\mathscr{T}$-injective by Proposition 2.3 (4). Thus $R^{1}(X / \mathscr{T}(X))=0$, and consequently $R^{1}(E(M) / M)=R^{1}(X)=0$ as desired.

$(2) \Rightarrow(3)$. Let $A$ be a $\mathscr{T}$-injective module, let $B \in \mathscr{F}$, and let $\theta: A \rightarrow B$ be an epimorphism. By (2) and the $\mathscr{T}$-injectivity of $A$, the sequence

$$
0=R^{1}(A) \longrightarrow R^{1}(B) \longrightarrow R^{2}(\operatorname{ker} \theta)=0
$$

is exact. Thus $R^{1},(B)=0$; so $B \in \mathscr{F}$ is $\mathscr{G}$-injective.

$(3) \Rightarrow(4)$ is trivial.

$(4) \Rightarrow(1)$. Let $D \in \mathscr{D} \mathscr{G}$, and let $\theta: D \rightarrow M$ be an epimorphism. Now $\theta$ naturally induces an epimorphism

$$
\bar{\theta}: D / \mathscr{S}(D) \longrightarrow M / \theta(\mathscr{G}(D)) \text {. }
$$

Since $D / \mathscr{T}(D)$ is $\mathscr{G}$-injective, then

$$
(M / \theta(\mathscr{G}(D))) /\left(\mathscr{G}(M) / \theta\left(\mathscr{G}^{-}(D)\right)\right) \cong M / \mathscr{G}(M) \in \mathscr{F}
$$

is also $\mathscr{T}$-injective by (4).

From the definition of $\mathscr{G}$-divisible, it follows that $\theta(\mathscr{G}(D)) \in \mathscr{C}_{\alpha}$ for some ordinal $\alpha$. By Lemma $1.1(3), \bar{\theta}^{-1}\left(\mathscr{G}\left(M / \theta\left(\mathscr{G}^{-}(D)\right)\right)\right)$ is $\mathscr{T}$ injective. Thus

$$
\mathscr{T}(M) / \theta(\mathscr{G}(D)) \cong \mathscr{T}^{-}(M / \theta(\mathscr{T}(D))) \in \mathscr{C}_{1},
$$

and consequently $\mathscr{S}^{-}(M) \in \mathscr{C}_{\alpha+1}$.

Corollary 2.6. The class $\mathscr{D} \mathscr{C}$ of -divisible modules is closed under homomorphic images.

Proof. This follows from Proposition 2.5 and [1, p. 197].

We shall say that the filter $F(\mathscr{G})$ is $\mathscr{G}$-noetherian if the following property holds: if $I_{1} \subset I_{2} \subset I_{3} \cdots$ is a countable ascending chain of left ideals whose union is in $F(\mathscr{T})$, then $\mathrm{I}_{n} \in F(\mathscr{T})$ for some $n$. The reader is directed to [8] and [15] for an extensive discussion of the $\mathscr{F}$-noetherian property. Our next proposition adds another equivalent condition to the lists given in [8, Theorem 4.4] and [15, Prop. 12.1]. 
Proposition 2.7. $\mathscr{D} \mathscr{T}$ is closed under direct sums if and only if $F(\mathscr{T})$ is $\mathscr{T}$-noetherian.

Proof. Let $\mathscr{A}$ be an index set, and let $A_{\alpha} \in \mathscr{D} \mathscr{T}$ for each $\alpha \in \mathscr{A}$. Since $\mathscr{T}\left(\bigoplus \sum_{\alpha \in \mathscr{A}} A_{\alpha}\right)=\bigoplus \sum_{\alpha \varepsilon_{\mathscr{N}}} \mathscr{T}\left(A_{\alpha}\right)$, it follows that $\mathscr{T}\left(\bigoplus \sum_{\alpha \in, .} A_{\alpha}\right) \in$ $\mathscr{C}_{\mu}$, where $\mu=\sup \left\{\beta_{\alpha} \mid \mathscr{T}\left(A_{\alpha}\right) \in \mathscr{E}_{\beta_{\alpha}}\right\}$. Hence $\mathscr{D} \mathscr{T}$ is closed under direct sums if and only if $\left(\bigoplus \sum_{\alpha \epsilon \mathscr{}} A_{\alpha}\right) / \mathscr{T}\left(\bigoplus \sum_{\alpha \in \mathscr{A}} A_{\alpha}\right) \cong \bigoplus \sum_{\alpha \epsilon, \mho}\left(A_{\alpha} / \mathscr{T}\left(A_{\alpha}\right)\right)$ is $\mathscr{T}$-injective. But the latter condition holds if and only if any direct sum of $\mathscr{T}$-injective modules in $\mathscr{F}$ is $\mathscr{T}$-injective. So the result follows from [8, Theorem 4.4].

COROLlaRY 2.8. $\mathscr{D C}$ is closed under direct sums if and only if $F(\mathscr{C})$ has a cofinal subset of finitely generated left ideals. In particular, if $R$ has a semi-simple maximal left quotient ring, then $\mathscr{D C}$ is closed under direct sums.

REMARK. Corollary 2.8 adds another condition to the list given in [16, Theorem 2.1].

COROLlaRY 2.9. If $F(\mathscr{G})$ has a cofinal subset of finitely generated left ideals, then every module $A$ has a (necessarily unique) largest submodule $\mathscr{D} \mathscr{G}(A)$ in $\mathscr{D} \mathscr{G}$. In particular, if $R$ has a semi-simple maximal left quotient ring, then every module $A$ has a largest submodule $\mathscr{D} \mathscr{G}(A)$ in $\mathscr{D} \mathscr{G}$.

Proof. This follows from Proposition 2.4 (4), Corollary 2.6, Corollary 2.8, and [4, Theorem 2.3].

3. Analogs of the Dedekind domain case. A commutative integral domain $D$ is called Dedekind if any one of the following three equivalent properties hold: (a) every ideal of $D$ is projective; (b) every divisible module is injective; and (c) the homomorphic image of an injective module is injective. In this section, we look at the analogs of these conditions in terms of hereditary torsion theories $(\mathscr{T}, \mathscr{F}$ ) over more general rings: (a) every left ideal in $F(\mathscr{T})$ is projective; (b) every $\mathscr{T}$-divisible module is $\mathscr{T}$-injective; and (c) the homomorphic image of a $\mathscr{T}$-injective module is $\mathscr{T}$-injective. Our goal is to obtain characterizations of these conditions in terms of the class $\mathscr{D} \mathscr{T}$ of $\mathscr{T}$-divisible modules.

We start our investigation by considering the following condition: h.d. $E_{\digamma}(F) \leqq 1$ for all free modules $F \in_{R} \mathscr{L C}$.

Clearly any torsion theory over a left hereditary ring satisfies (*). In the commutative integral domain case, Matlis [11] and [12] considered 
rings for which h.d. $Q \leqq 1$, where $Q$ is the quotient field of the domain. Since $Q=Q_{s}$, the following result shows that $\left(^{*}\right)$ is a "natural" generalization of the condition, "h.d. $Q \leqq 1 . "$

Proposition 3.1. Let $R \in \mathscr{F}$, and let $F(\mathscr{T})$ be $\mathscr{T}$-noetherian. Then (*) holds if and only if h.d. $Q, \leqq 1$.

Proof. The "only if" part is trivial since $Q_{,}=E_{-}(R)$.

Let $\mathscr{A}$ be an index set, let $R^{(\alpha)} \cong R$ for each $\alpha \in \mathscr{A}$, and let $F=\bigoplus \sum_{\alpha \epsilon,}, R^{(\alpha)}$. Since $F(\mathscr{T})$ is $\mathscr{T}$-noetherian, then $E_{\mathscr{J}}(F)=$ $\bigoplus \sum_{\alpha \in, Q^{(\alpha)}}$, where $Q^{(\alpha)} \cong Q$, for each $\alpha \in \mathscr{A}$. So if h.d. $Q$, then

$\operatorname{Ext}^{2}(E,(F), \ldots)=\operatorname{Ext}^{2}\left(\bigoplus \sum_{\alpha \in \mathscr{S}} Q_{(\alpha)}^{(\alpha)},\right) \cong \Pi_{\alpha \epsilon \mathscr{S}} \operatorname{Ext}\left(Q_{\zeta}^{(\alpha)},-\right)=0$.

Hence h.d. $E_{J}(F) \leqq 1$.

LEMma 3.2. If every homomorphic image of a $\mathscr{T}$-injective module is $\mathscr{T}$-injective, then $\left(^{*}\right)$ holds.

Proof. Let $A$ be a $\mathscr{T}$-injective module, let $F$ be a free module, and let $L=E,(F) / F \in \mathscr{T}$. By the hypothesis, the sequence

$$
0=\operatorname{Ext}^{1}(L, E(A) / A) \longrightarrow \operatorname{Ext}^{2}(L, A) \longrightarrow \operatorname{Ext}^{2}(L, E(A))=0
$$

is exact. Hence $\operatorname{Ext}^{2}(L, A)=0$.

Now let $M \in_{R} \mathscr{M}$, and set $A=E,(M)$. By the hypothesis and the preceding paragraph, the sequence

$$
0=\operatorname{Ext}^{1}(L, A / M) \longrightarrow \operatorname{Ext}^{2}(L, M) \longrightarrow \operatorname{Ext}^{2}(L, A)=0
$$

is exact. Hence $\operatorname{Ext}^{2}(L, M)=0$. Since $F$ is a free module, the sequence

$$
0=\operatorname{Ext}^{2}(L, M) \longrightarrow \operatorname{Ext}^{2}\left(E_{J}(F), M\right) \longrightarrow \operatorname{Ext}^{2}(F, M)=0
$$

is exact. Thus $\operatorname{Ext}^{2}\left(E_{\mathscr{\sigma}}(F), M\right)=0$ for all $M \in_{R} \mathscr{C l}$, and hence (*) holds.

Lemma 3.3. If $\left(^{*}\right)$ holds, then the following statements are equivalent.

(1) $\operatorname{Ext}^{1}\left(E_{\sigma}(F) / F, H\right)=0$ for all free modules $F$.

(2) Every homomorphism from a free module $F$ into $H$ can be extended to a homomorphism from $E_{\Im}(F)$ into $H$.

(3) $H$ is a homomorphic image of a $\mathscr{T}$-injective module.

Proof. $\quad(1) \Rightarrow(2)$ follows from the exact sequence $\operatorname{Hom}\left(E_{丂}(F), H\right) \longrightarrow \operatorname{Hom}(F, H) \rightarrow \operatorname{Ext}^{1}\left(E_{Y}(F) / F, H\right)$. 
$(2) \Rightarrow(3)$ is trivial since every module is a homomorphic image of a free module.

(3) $\Rightarrow(1)$. Let $A$ be a $\mathscr{T}$-injective module, and let $\theta: A \rightarrow H$ be an epimorphism. It follows from $(*)$ that h.d. $E_{\mathscr{J}}(F) / F \leqq 1$ for any free module $F$. Using this fact, we have the exact sequence

$$
\begin{aligned}
\operatorname{Ext}^{1}\left(E_{J}(F) / F, A\right) & \longrightarrow \operatorname{Ext}^{1}\left(E_{J}(F) / F, H\right) \\
& \longrightarrow \operatorname{Ext}^{2}\left(E_{J}(F) / F, \operatorname{ker} \theta\right)=0 .
\end{aligned}
$$

Since $A$ is $\mathscr{T}$-injective, the left end of this sequence is also 0 , and hence (1) follows from the exactness of the sequence.

REMARK. Lemma 3.3 is a generalization of [11, Prop. 2.1] (see also [13, Prop. 3.1]). If $R \in \mathscr{F}$ and $F(\mathscr{T})$ is $\mathscr{T}$-noetherian, then condition (2) in Lemma 3.3 can be replaced by the following condition: every homomorphism from $R$ into $H$ can be extended to a homomorphism from $Q_{\sigma}$ into $H$.

The next lemma gives us conditions which are sufficient to insure that every $\mathscr{T}$-divisible module is a homomorphic image of a $\mathscr{T}$-injective module.

LEMma 3.4. If $R \in \mathscr{F}$, if $F(\mathscr{T})$ is $\mathscr{T}$-noetherian, and if (*) holds, then every $\mathscr{T}$-divisible module is a homomorphic image of a $\mathscr{T}$-injective module.

Proof. Let $D \in \mathscr{D} \mathscr{T}$; so $D / \mathscr{T}(D)$ is $\mathscr{T}$-injective. Temporarily assume that $\mathscr{T}(D)$ is a homomorphic image of a $\mathscr{T}$-injective module. Then by Lemma 3.3, the sequence

$$
\begin{aligned}
0=\operatorname{Ext}^{1}\left(E_{\mathscr{S}}(F) / F, \mathscr{T}(D)\right) & \longrightarrow \operatorname{Ext}^{1}\left(E_{-}(F) / F, D\right) \\
& \longrightarrow \operatorname{Ext}^{1}\left(E_{-}(F) / F, D / \mathscr{T}(D)\right)=0
\end{aligned}
$$

is exact for every free module $F$. Hence $\operatorname{Ext}^{1}\left(E_{-}(F) / F, D\right)=0$ by exactness. Thus Lemma 3.3 implies $D$ is a homomorphic image of a $\mathscr{T}$-injective module. So in order to prove the theorem, it is sufficient to prove that $\mathscr{T}(D)$ is a homomorphic image of a $\mathscr{T}$-injective module.

Since $D \in \mathscr{D} \mathscr{T}$, then $\mathscr{T}(D) \in \mathscr{C}_{\alpha}$ for some ordinal $\alpha$. Let $\left\{\mathscr{D}_{\beta} \mid \beta \in \mathscr{B}\right\}$ be the set of submodules of $\mathscr{T}(\mathscr{D})$ such that each $D_{\beta}$ is a homomorphic image of a direct sum of $\mathscr{T}$-injective modules. It is easy to see that there exists $\gamma \in \mathscr{B}$ such that $D_{r}=\sum_{\beta \in \mathscr{B}} D_{\beta}$. Now each $\mathscr{T}$-injective module is a homomorphic image of $E_{\mathscr{J}}(F)$ for some free module $F$. Since $F(\mathscr{T})$ is a $\mathscr{T}$-noetherian, then by [8, Theorem 4.4] each $E_{J}(F)$ is a direct sum of copies of $Q_{\supset}$, and any direct sum of copies of $Q_{\sigma}$ is $\mathscr{T}$-injective. Hence $D_{\gamma}$ is a homomorphic 
image of a $\mathscr{T}$-injective module. To complete the proof, we wish to show $D_{r}=\mathscr{T}(D)$.

If $D_{\gamma} \neq \mathscr{T}(D) \in \mathscr{C}_{\alpha}$, then there exists a $\mathscr{T}$-injective module $A$ such that $\operatorname{Hom}\left(A, \mathscr{T}(D) / D_{r}\right) \neq 0$. Let $\phi: A \rightarrow \mathscr{T}(D) / D_{r}$ be a nonzero homomorphism, and let im $\phi=B / D_{r}$, where $B \subseteq \mathscr{T}(D)$. By Lemma 3.3 , the sequence

$$
\begin{aligned}
0=\operatorname{Ext}^{1}\left(E_{-}(F) / F, D_{\gamma}\right) & \longrightarrow \operatorname{Ext}^{1}\left(E_{-}(F) / F, B\right) \\
& \longrightarrow \operatorname{Ext}^{1}\left(E_{-}(F) / F, B / D_{\gamma}\right)=0
\end{aligned}
$$

is exact. Hence $\operatorname{Ext}^{1}(E,(F) / F, B)=0$; so $B$ is a homomorphic image of a $\mathscr{T}$-injective module by Lemma 3.3. Hence there exists $\sigma \in \mathscr{P}$ such that $B=D_{\sigma} \subseteq \sum_{\beta \in \mathscr{B}} D_{\beta}=D_{r}$. But this contradicts our choice of $B$; so $D_{r}=\mathscr{T}(D)$ as desired.

Proposition 3.5. Suppose that $R \in \mathscr{F}$ and that $F(\mathscr{T})$ is $\mathscr{T}=$ noetherian. If every homomorphic image of a $\mathscr{T}$-injective module is $\mathscr{T}$-injective, then every homomorphic image of a $\mathscr{T}$-divisible module is $\mathscr{T}$-injective.

Proof. By Lemma 3.2 and the hypothesis, (*) holds. Since $F(\mathscr{T})$ is $\mathscr{T}$-noetherian, the Lemma 3.4 implies every $\mathscr{T}$-divisible is a homomorphic image of a $\mathscr{T}$-injective module. Consequently, every homomorphic image of a $\mathscr{T}$-divisible module is also a homomorphic image of a $\mathscr{T}$-injective module; so the result follows from the hypothesis.

Proposition 3.6. Suppose that $\mathscr{T}$ is stable. If every homomorphic image of a $\mathscr{T}$-divisible module is $\mathscr{T}$-injective, then every homomorphic image of a $\mathscr{T}$-injective module is $\mathscr{T}$-injective.

Proof. Since $\mathscr{T}$ is stable, Proposition 2.1 (2) implies that every $\mathscr{T}$-injective module is $\mathscr{T}$-divisible; so the result now follows from the hypothesis.

Collecting our previous results, we obtain:

THeorem 3.7 Suppose that $\mathscr{T}$ is stable and that $F(\mathscr{T})$ is $\mathscr{T}$ noetherian. If $R \in \mathscr{F}$, then the following statements are equivalent.

(1) Every homomorphic image of a $\mathscr{T}$-divisible module is $\mathscr{T}$ injective.

(2) Every homomorphic image of a $\mathscr{T}$-injective module is $\mathscr{T}$ injective.

(3) Every left ideal in $F(\mathscr{T})$ is projective.

Proof. $(1) \Rightarrow(2)$ is immediate from Proposition $3.6 ;(2) \Rightarrow(1)$ is immediate from Proposition 3.5; and $(2) \Leftrightarrow(3)$ is [9, Prop. 3.3]. 
Corollary 3.8. Suppose that $R$ has a semi-simple left maximal quotient ring. Then every homomorphic image of a $\mathscr{G}$-divisible module is injective if and only if $R$ is hereditary.

As a special case of Corollary 3.8, we obtain the following characterization of a Dedekind domain.

CoRollary 3.9. Let $R$ be a commutative integral domain. Then every homomorphic image of a G-divisible module is injective if and only if $R$ is a Dedekind domain.

Remarks. From Corollary 3.9 we see that if $R$ is a Dedekind domain, then every $\mathscr{G}$-divisible module is injective, and hence every divisible module is $\mathscr{C}$-divisible. However, the classes of divisible and $\mathscr{G}$-divisible modules may coincide without $R$ being a Dedekind domain. In particular, if $R$ is a commutative integral domain with quotient field $Q$ that is a countably generated $R$-module, then every divisible module is $\mathscr{C}$-divisible by [11, Theorem 1.3] and Corollary 2.6.

THEOREM 3.10. If $\mathscr{T}$ is stable, then the following statements are equivalent.

(1) A module is $\mathscr{T}$-divisible if and only if it is $\mathscr{T}$-injective.

(2) Every $\mathscr{T}$-divisible module is $\mathscr{T}$-injective.

(3) Every $\mathscr{T}$-divisible module in $\mathscr{T}$ is injective.

Moreover, each of the above three statements implies that the following statements are true.

(a) $F(\mathscr{T})$ satisfies the ascending chain condition.

(b) $\operatorname{inj} \operatorname{dim} M \leqq 1$ whenever $E(M) / M \in \mathscr{T}$; in particular, inj $\operatorname{dim} T \leqq 1$ for all $T \in \mathscr{T}$.

Proof. $(1) \Rightarrow(2)$ is trivial.

(2) $\Rightarrow(3)$. Let $T \in \mathscr{T} \cap \mathscr{D} \mathscr{T} . \quad$ By (2), $T$ is $\mathscr{T}$-injective. Since $\mathscr{T}$ is stable, Lemma 1.1 (4) implies $T$ is injective.

$(3) \Rightarrow(1)$. Since $\mathscr{T}$ is stable, any $\mathscr{T}$-injective module is $\mathscr{T}$-divisible by Proposition 2.3 (2). So we need to show that any $\mathscr{T}$-divisible module is $\mathscr{T}$-injective. Let $D \in \mathscr{D} \mathscr{T}$; then $D / \mathscr{T}(D)$ is $\mathscr{T}$-injective. Since $D \in \mathscr{D} \mathscr{T}$, then also $\mathscr{T}(D) \in \mathscr{D} \mathscr{T}$; so $\mathscr{T}(D)$ is injective by (3). Therefore $D \cong \mathscr{T}(D) \oplus(D / \mathscr{T}(D))$ is $\mathscr{T}$-injective.

(3) $\Rightarrow$ (a). Let $I_{1} \subseteq I_{2} \subseteq I_{3} \subseteq \cdots$ be an ascending chain with each $I_{n} \in F(\mathscr{T})$. Since $\mathscr{T}$ is stable, each $E\left(R / I_{n}\right) \in \mathscr{T}$, and hence $\bigoplus \sum_{n=1}^{\infty} E\left(R / I_{n}\right) \in \mathscr{T}$. Now any direct sum of injective modules in $\mathscr{T}$ is $\mathscr{T}$-divisible and hence injective by (3). Therefore $\bigoplus \sum_{n=1}^{\infty} E\left(R / I_{n}\right)$ is injective. We now can complete the proof of (a) by a standard argument. Let $I=\bigcup_{n=1}^{\infty} I_{n}$, and define $\theta: I \rightarrow \bigoplus \sum_{n=1}^{\infty} E\left(R / I_{n}\right)$ via $x \rightarrow$ $\left(x+I_{n}\right)$ for all $x \in I$. By injectivity, $\theta$ extends to a map from $R$ to 
$\bigoplus \sum_{n=1}^{\infty} E\left(R / I_{n}\right)$. Since $R$ has an identity element, it follows that $I=I_{m}$ for some integer $m$.

(3) $\Rightarrow(\mathrm{b})$. If $E(M) / M \in \mathscr{T}$, then $E(M) / M$ is injective by (3). So the sequence

$$
0=\operatorname{Ext}^{1}\left({ }_{-}, E(M) / M\right) \rightarrow \operatorname{Ext}^{2}\left(_{-}, M\right) \rightarrow \operatorname{Ext}^{2}\left({ }_{-}, E(M)\right)=0 .
$$

is exact, and hence $\operatorname{Ext}^{2}(, M)=0$. Therefore, $\operatorname{inj} \operatorname{dim} M \leqq 1$.

CoROLlary 3.11. The following statements are equivalent.

(1) A module is $\mathscr{G}$-divisible if and only if it is injective.

(2) Every $\mathscr{G}$-divisible module is injective.

(3) Every $\mathscr{G}$-divisible module in $\mathscr{G}$ is injective.

(4) $F(\mathscr{G})$ satisfies the ascending chain condition, and l. gl. $\operatorname{dim} R \leqq 1$.

Proof. (1) $\Leftrightarrow(2) \Leftrightarrow(3) \Rightarrow(4)$ is immediate from Theorem 3.10. $(4) \Rightarrow(3)$. Let $G \in \mathscr{G} \cap \mathscr{D} \mathscr{G}$. Let $\left\{A_{\alpha} \mid \alpha \in \mathscr{A}\right\}$ be the set of submodules of $G$ which are homomorphic images of injective modules. Set $A=\sum_{\alpha \in \oiint} A_{\alpha}$. If $\theta_{\alpha}: E_{\alpha} \rightarrow A_{\alpha}$ is an epimorphism of an injective module $E_{\alpha}$, then by (4) $A_{\alpha}$ is injective. We wish to show that $E=\bigoplus \sum_{\alpha \in S_{t}} A_{\alpha}$ is injective.

To see $E$ is injective, it is sufficient to show that, for each essential left ideal $I$ of $R$, any homomorphism $\varphi: I \rightarrow E$ can be extended to a homomorphism $\varphi^{\prime}: R \rightarrow E$. Since $E \in \mathscr{G}$, then $I / \operatorname{ker} \varphi \in \mathscr{G}$; so since $I \in F(\mathscr{G})$, it follows that $\operatorname{ker} \varphi \in F(\mathscr{G})$ also. Hence $R / \operatorname{ker} \varphi$ has a.c.c. on submodules by (4). But then $I / \operatorname{ker} \varphi$ is finitely generated, and hence im $\varphi \subseteq \bigoplus \sum_{\alpha \in \mathscr{B}} A_{\alpha}$, where $\mathscr{B}$ is a finite subset of $\mathscr{A}$. Since a direct sum of finitely many injective modules is injective, then $\varphi$ can be extended to $\phi^{\prime}: R \rightarrow \bigoplus \sum_{\alpha \in \mathscr{B}} A_{\alpha} \subseteq E$.

Now $E$ is injective; so $A$ is also injective by (4). Hence $G=A \oplus X$ for some $X \subseteq G$. Since $X \in \mathscr{G}$ is $\mathscr{G}$-divisible by Proposition 2.4 (3), then $X=0$ by the construction of $A$ and the definition of $\mathscr{G}$-divisible. Hence $G=A$ is injective.

An immediate consequence of Corollary 3.11 is the following characterization of a Dedekind domain.

CoROllary 3.12. Let $R$ be a commutative integral domain. Every $\mathscr{G}$-divisible module is injective if and only if $R$ is a Dedekind domain.

4. The splitting of $\mathscr{T}$-divisible modules and h.d. $Q_{5} \leqq 1$. In this section we show that, for a wide class of torsion theories with $R \in \mathscr{F}$, h.d. $Q_{\mathscr{F}} \leqq 1$ if and only if every $\mathscr{T}$-divisible module splits.

We start by considering the condition:

(I) If $F$ is a free module and $A$ is $\mathscr{T}$-injective, then 


$$
\operatorname{Ext}^{2}\left(E_{\sigma}(F), A\right)=0 .
$$

For the Goldie torsion theory, the class of injective modules coincides with the class of $\mathscr{C}$-injective modules; so (I) always holds for the Goldie torsion theory. The first result of this section relates (I) to $Q_{\mathscr{S}}$ when $R \in \mathscr{F}$.

Proposition 4.1. If $R \in \mathscr{F}$ and if $F(\mathscr{T})$ is $\mathscr{T}$-noetherian, then (I) holds if and only if $\operatorname{Ext}^{2}\left(\mathrm{Q}_{-}, A\right)=0$ for all $\mathscr{T}$-injective modules $A$.

Proof. Since $Q_{\nearrow}=E_{\mathscr{T}}(R)$, the "only if" part is trivial. Since $F(\mathscr{T})$ is $\mathscr{T}$-noetherian, $E_{\mathscr{T}}(F) \cong \bigoplus \sum_{\alpha \in \Theta} Q_{J^{(\alpha)}}$ for any free module $F\left(Q_{\mathscr{T}}{ }^{(\alpha)} \cong Q_{\mathscr{Y}}\right.$ for all $\left.\alpha \in \mathscr{A}\right)$. So if $\operatorname{Ext}^{2}\left(Q_{J}, A\right)=0$ for all $\mathscr{T}$-injective modules $A$, then

$$
\operatorname{Ext}^{2}\left(E_{\mathscr{S}}(F), A\right) \cong \operatorname{Ext}^{2}\left(\bigoplus \sum_{\alpha \in \mathscr{A}} Q_{\mathscr{S}}^{(\alpha)}, A\right) \cong \Pi_{\alpha \in \mathscr{A}} \operatorname{Ext}^{2}\left(Q_{\mathscr{S}^{(\alpha)}}, A\right)=0 .
$$

The following result generalizes [11, Theorem 1.2] (see also [13, Prop. 2.2]).

Lemma 4.2. Assume that (I) holds and that $R \in \mathscr{F}$. Suppose that any $\mathscr{T}$-divisible module $D$ splits whenever $\mathscr{T}(D)$ is a homomorphic image of a $\mathscr{T}$-injective module. Then $\left(^{*}\right)$ holds; i.e., h.d. $E_{\mathcal{J}}(F) \leqq 1$ for all free modules $F \in_{R} \mathscr{M l}$.

Proof. Let $A \in_{R} \mathscr{C l}$, and let $F$ be a free module. Then consider the exact sequence

$$
0 \longrightarrow E_{\mathscr{T}}(A) / A \stackrel{\alpha}{\longrightarrow} D \longrightarrow E_{j}(F) \longrightarrow 0 \text {. }
$$

Since $\mathscr{T}$ is closed under homomorphic images and since $E_{\mathscr{J}}(F) \in \mathscr{F}$, it follows that im $\alpha=\mathscr{T}(D)$. Since $\mathscr{T}(D) \cong E_{,}(A) / A$ and $E,(F) \in \mathscr{F}$, then $D$ is $\mathscr{T}$-divisible. By hypothesis $\mathscr{T}^{-}(D)$ is a direct summand of $D$. But then the above sequence must split because im $\alpha=\mathscr{T}(D)$; thus $\operatorname{Ext}^{1}\left(E_{\digamma}(E), E_{\nearrow}(A) / A\right)=0$. So by (I), the sequence

$$
\begin{aligned}
0=\operatorname{Ext}^{1}\left(E_{\digamma}(F), E_{\nearrow}(A) / A\right) & \longrightarrow \operatorname{Ext}^{2}\left(E_{\nearrow}(F), A\right) \\
& \operatorname{Ext}^{2}\left(E_{\nearrow}(F), E_{\nearrow}(A)\right)=0
\end{aligned}
$$

is exact. From the exactness it follows that $\operatorname{Ext}^{2}\left(E_{\sigma}(F), A\right)=0$, and hence h.d. $E_{J}(F) \leqq 1$.

Next we need the following condition:

(II) A $\mathscr{T}$-divisible module splits if it is a homomorphic image of a $\mathscr{T}$-injective module.

Matlis [11, Theorem 1.1] showed that (II) holds for the usual torsion theory over a commutative integral domain. Armendariz [2, Theorem 2.5] extended Matlis' result by showing that the Goldie tor- 
sion submodule of a homomorphic image of a quasi-injective module splits off. (Armendariz's result is for any ring.) A consequence of Armendariz's result is that (II) always holds for Goldie's torsion theory.

Lemma 4.3. Assume $\left(^{*}\right)$ and (II) hold. Let $D$ be a $\mathscr{S}$-divisible module, and let $H$ be a homomorphic image of a 5 -injective module such that $H \subseteq \mathscr{S}^{-}(D)$. Then $D$ splits if and only if $D / H$ splits.

Proof. If $D$ splits, then $D=\mathscr{G}(D) \oplus S$, where $S \in \mathscr{F}$. Then $D / H \cong(\mathscr{J}(D) / H) \oplus S$. Since $\mathscr{S}(D) / H=\mathscr{J}(D / H)$, then $D / H$ splits.

Conversely, if $D / H$ splits, then $D / H=(\mathscr{S}(D) / H) \oplus(G / H)$, where $H \cong G \cong D$. Since $D$ is $\mathscr{T}$-divisible, then $G / H \cong(D / H) /(\mathscr{T}(D) / H) \cong$ $D / \mathscr{T}(D) \in \mathscr{F}$ is $\mathscr{S}$-injective. Thus $\left(^{*}\right)$ and Lemma 3.3 imply that the sequence

$$
0=\operatorname{Ext}^{1}(E(F) / F, H) \rightarrow \operatorname{Ext}^{1}(E,(F) / F, G) \rightarrow \operatorname{Ext}^{1}(E,(F) / F, G / H)=0
$$

is exact. Hence $\operatorname{Ext}^{1}\left(E_{,}(F) / F, G\right)=0$. By Lemma 3.3, $G$ is a homomorphic image of a $\mathscr{T}$-injective module. Since $G / H \in \mathscr{F}$ is $\mathscr{T}$ injective, the definition of $H$ enables us to see that $G$ is $\mathscr{T}$-divisible. By (II) $G$ splits; thus $G=H \oplus L$ (as $H=\mathscr{T}(G)$ ). Hence $D=$ $\mathscr{T}(D) \oplus L$, i.e., $D$ splits.

Theorem 4.4. Assume (I) and (II) hold. If $F(\mathscr{G})$ is G-noetherian and $R \in \mathscr{F}$, then the following statements are equivalent.

(1) h.d. $Q . \leqq 1$.

(2) Every G-divisible module splits.

Proof. $\quad(1) \Rightarrow(2)$. By Proposition 3.1, (*) holds; so by Lemma 3.4, every $\mathscr{T}$-divisible module $D$ is a homomorphic image of a $\mathscr{T}$-injective module. Thus $\mathscr{T}(D)$ is also a homomorphic image of a $\mathscr{T}$-injective module by Lemma 1.1 (3). So it follows trivially from Lemma 4.3 that $D$ splits.

$(2) \Rightarrow(1)$ follows immediately from Lemma 4.2 and Proposition 3.1.

As an immediate consequence of Theorem 4.4, we have the following result.

COROLlary 4.5. If $R$ has a semi-simple maximal left quotient ring, then the following statements are equivalent.

(1) h.d. $E(R) \leqq 1$.

(2) Every C-divisible module splits.

A special case of this result is the following corollary which the 
reader may wish to compare with [11, Cor. 2.6] (see also [13, Prop. 4.2]).

COROLlary 4.6 If $R$ is an integral domain with quotient field $Q \neq R$, then the following statements are equivalent.

(1) h.d. $Q=1$.

(2) Every $\mathscr{G}$-divisible module splits.

Finally, we note that Corollary 4.6 adds to the list given in [12, Theorem 10.1] of conditions equivalent to h.d. $Q=1$.

\section{REFERENCES}

1. J. S Alin and S. E. Dickson, Goldie's torsion theory and its derived functor, Pacific J. Math., 24 (1968), 195-203.

2. E. P. Armendariz, Quasi-injective modules and stable torsion classes, Pacific J. Math., 31 (1969), 277-280.

3. H. Cartan and S. Eilenberg, Homological Algebra, Princeton, 1956.

4. S. E. Dickson, A torsion theory for Abelian categories, Trans. Amer. Math. Soc., 121 (1966), 223-235.

5. — Direct Decompositions of Radicals, Proc. Conference on Categorical Algebra at LaJolla in 1965, Springer Verlag, 1966.

6. L. Fuchs, Torsion preradicals and ascending Loewy series of modules, J. Reine Angew. Math., 239-240 (1970), 169-179.

7. P. Gabriel, Des categories abéliennes, Bull. Soc. Math. France, 90 (1962), 323-448.

8. O. Goldman, Rings and modules of quotients, J. Algebra, 13 (1969), 10-47.

9. G. Helzer, On divisibility and injectivity, Canad. J. Math., 18 (1966), 901-919.

10. J. P. Jans, Some aspects of torsion, Pacific J. Math., 15 (1965), 1249-1259.

11. E. Matlis, Divisible modules, Proc. Amer. Math. Soc., 11 (9160), 385-391.

12. - Cotorsion modules, Memoirs Amer. Math. Soc., \#49, Providence, 1964.

13. M.A.R. Qureshi, Homomorphic images of an injective module, Quart. J. Math., Oxford, 22 (1971), 85-90.

14. D. Sanderson, A generalization of divisibility and injectivity in modules, Canad. Math. Bull., 8 (1965), 505-513.

15. B. Stenström, Rings and Modules of Quotients, Lecture Notes in Mathematics \#237, Springer-Verlag, 1971.

16. M. L. Teply, Some aspects of Goldie's torsion theory, Pacific J. Math., 29 (1969), 447-459.

17. Homological dimension and splitting torsion theories, Pacific J. Math., 34 (1970), 193-205.

Received February 28, 1972.

UNIVERSITY OF FLORIDA 


\section{PACIFIC JOURNAL OF MATHEMATICS}

\section{EDITORS}

\author{
H. SAMELson \\ Stanford University \\ Stanford, California 94305 \\ C. R. HоввY \\ University of Washington \\ Seattle, Washington 98105
}

J. DugundJI

Department of Mathematics

University of Southern California

Los Angeles, California 90007

RICHARD ARENS

University of California

Los Angeles, California 90024

\section{ASSOCIATE EDITORS}
E. F. BeCKENBACH
B. H. NEUMANN
F. WOLF
K. YoshidA

\section{SUPPORTING INSTITUTIONS}

UNIVERSITY OF BRITISH COLUMBIA

CALIFORNIA INSTITUTE OF TECHNOLOGY

UNIVERSITY OF CALIFORNIA

MONTANA STATE UNIVERSITY

UNIVERSITY OF NEVADA

NEW MEXICO STATE UNIVERSITY

OREGON STATE UNIVERSITY

UNIVERSITY OF OREGON

OSAKA UNIVERSITY

\author{
UNIVERSITY OF SOUTHERN CALIFORNIA \\ STANFORD UNIVERSITY \\ UNIVERSITY OF TOKYO \\ UNIVERSITY OF UTAH \\ WASHINGTON STATE UNIVERSITY \\ UNIVERSITY OF WASHINGTON

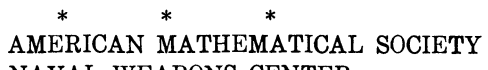 \\ NAVAL WEAPONS CENTER
}

The Supporting Institutions listed above contribute to the cost of publication of this Journal, but they are not owners or publishers and have no responsibility for its content or policies.

Mathematical papers intended for publication in the Pacific Journal of Mathematics should be in typed form or offset-reproduced, (not dittoed), double spaced with large margins. Underline Greek letters in red, German in green, and script in blue. The first paragraph or two must be capable of being used separately as a synopsis of the entire paper. The editorial "we" must not be used in the synopsis, and items of the bibliography should not be cited there unless absolutely necessary, in which case they must be identified by author and Journal, rather than by item number. Manuscripts, in duplicate if possible, may be sent to any one of the four editors. Please classify according to the scheme of Math. Rev. Index to Vol. 39. All other communications to the editors should be addressed to the managing editor, Richard Arens, University of California, Los Angeles, California, 90024.

50 reprints are provided free for each article; additional copies may be obtained at cost in multiples of 50 .

The Pacific Journal of Mathematics is issued monthly as of January 1966. Regular subscription rate: $\$ 48.00$ a year (6 Vols., 12 issues). Special rate: $\$ 24.00$ a year to individual members of supporting institutions.

Subscriptions, orders for back numbers, and changes of address should be sent to Pacific Journal of Mathematics, 103 Highland Boulevard, Berkeley, California, 94708.

PUBLISHED BY PACIFIC JOURNAL OF MATHEMATICS, A NON-PROFIT CORPORATION

Printed at Kokusai Bunken Insatsusha (International Academic Printing Co., Ltd.), 270, 3-chome Totsuka-cho, Shinjuku-ku, Tokyo 160, Japan. 


\section{Pacific Journal of Mathematics}

\section{Vol. 45, No. $2 \quad$ October, 1973}

Kenneth Paul Baclawski and Kenneth Kapp, Induced topologies for quasigroups and loops ............................................. 393

D. G. Bourgin, Fixed point and $\min -\max$ theorems $\ldots \ldots \ldots \ldots \ldots \ldots \ldots$

J. L. Brenner, Zolotarev's theorem on the Legendre symbol ............... 413

Jospeh Atkins Childress, Jr., Restricting isotopies of spheres .............. 415

John Edward Coury, Some results on lacunary Walsh series ................ 419

James B. Derr and N. P. Mukherjee, Generalized Sylow tower groups. II . . . . . . 427

Paul Frazier Duvall, Jr., Peter Fletcher and Robert Allen McCoy, Isotopy Galois

spaces .......................................... 435

Mary Rodriguez Embry, Strictly cyclic operator algebras on a Banach space ... 443

Abi (Abiadbollah) Fattahi, On generalizations of Sylow tower groups ......... 453

Burton I. Fein and Murray M. Schacher, Maximal subfields of tensor products . . 479

Ervin Fried and J. Sichler, Homomorphisms of commutative rings with unit

element .......................................... 485

Kenneth R. Goodearl, Essential products of nonsingular rings ............. 493

George Grätzer, Bjarni Jónsson and H. Lakser, The amalgamation property in

equational classes of modular lattices ...........................

507

$\mathrm{H}$. Groemer, On some mean values associated with a randomly selected simplex

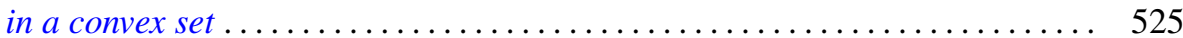

Marcel Herzog, Central 2-Sylow intersections .................... 535

Joel Saul Hillel, On the number of type-k translation-invariant groups ........ 539

Ronald Brian Kirk, A note on the Mackey topology for $\left(C^{b}(X)^{*}, C^{b}(X)\right) \ldots \ldots .543$

J. W. Lea, The peripherality of irreducible elements of lattice.............. 555

John Stewart Locker, Self-adjointness for multi-point differential operators ..... 561

Robert Patrick Martineau, Splitting of group representations ............... 571

Robert Massagli, On a new radical in a topological ring ................. 577

James Murdoch McPherson, Wild arcs in three-space. I. Families of Fox-Artin

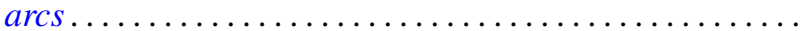

585

James Murdoch McPherson, Wild arcs in three-space. III. An invariant of

oriented local type for exceptional arcs . . . . . . . . . . . . ............ 599

Fred Richman, The constructive theory of countable abelian p-groups ........ 621

Edward Barry Saff and J. L. Walsh, On the convergence of rational functions

which interpolate in the roots of unity ..................

Harold Eugene Schlais, Non-aposyndesis and non-hereditary

decomposability..................................... 643

Mark Lawrence Teply, A class of divisible modules................... 653

Edward Joseph Tully, Jr., H-commutative semigroups in which each

homomorphism is uniquely determined by its kernel ................. 669

Garth William Warner, Jr., Zeta functions on the real general linear group ...... 681

Keith Yale, Cocyles with range $\{ \pm 1\} \ldots \ldots \ldots \ldots \ldots \ldots \ldots \ldots \ldots \ldots \ldots \ldots \ldots . \ldots \ldots$

Chi-Lin Yen, On the rest points of a nonlinear nonexpansive semigroup ........ 699 